\title{
Estimation of interfacial acidity of sodium dodecyl sulfate micelles
}

\author{
ARGHYA DEY and G NARESH PATWARI* \\ Department of Chemistry, Indian Institute of Technology Bombay, Powai, Mumbai 400076, India \\ e-mail: naresh@chem.iitb.ac.in
}

\begin{abstract}
An enhancement in the excited state proton transfer (ESPT) processes of coumarin-102 (C-102) dye was observed upon addition of salicylic acid and hydrochloric acid in sodium dodecyl sulfate (SDS) micellar solution. The phenomenon was observed only in the micellar medium of anionic surfactant SDS and not in case of cationic (CTAB) or neutral (Trition X -100) surfactants. ESPT of C-102 was also observed in aqueous solutions but on addition of very high concentrations of hydrochloric acid. However, on comparing the ratio of the protonated species from the emission spectra in the presence and absence of SDS micelle, a conclusive estimation of the local proton concentration at the Stern layer of SDS micelles could be evaluated.
\end{abstract}

Keywords. Coumarin 102; SDS micelles; ESPT.

\section{Introduction}

Excited state protonation and proton transfer reactions have been a subject of intense interest due to their potential applications in a variety of systems, such as fluorescent anion sensors and proton lasers. ${ }^{1}$ Excited state proton transfer (ESPT) occurs in a molecule, whenever there is large change in the acidity/basicity upon photoexcitation. Both inter- and intra-molecular versions of ESPT have been observed. For example, the proton transfer of 6-hydroxyquinolinium and $\mathrm{N}$ methyl-6-hydroxyquinolinium cations to the solvent is well-studied in water and in mixed solvents, ${ }^{2}$ reveals that in the excited state the hydroxyl group was found to behave as a superacid with ultrafast deprotonation rates. Apart from neat solutions, both aqueous and non-aqueous ESPT investigations have been carried out on various molecules in micro-heterogeneous media such as micelles, ${ }^{3}$ cyclodextrins ${ }^{4}$ and polymer-micelle aggregates. ${ }^{5}$ For instance, it has been observed that the ESPT reaction in 2-(2'-pyridyl)benzimidazole is enhanced in the presence of SDS micelles, ${ }^{6}$ which was attributed to the increased local acidity at the micellewater interface. Recent observations on excited state intramolecular proton transfer of $4^{\prime}-N, N$-diethylamino3-hydroxyflavone in room temperature ionic liquids have shown that solvation dynamics plays an important role in proton transfer mechanism. ${ }^{7}$

\footnotetext{
${ }^{*}$ For correspondence
}

Coumarin dyes and its derivatives have been extensively investigated, ${ }^{8}$ as fluorescent probes for various spectroscopic investigations, such as, energy transfer, ${ }^{9}$ solvation dynamics, ${ }^{10}$ fluorescence sensing ${ }^{11}$ and a host of others. 7-Aminocoumarins, such as coumarin-1, which is structurally very similar to C-102, are reported to exhibit a non-emissive intramolecular charge transfer (ICT) state in solvents of very high polarity. ${ }^{12}$ Decrease in fluorescence quantum yield with increasing solvent polarity has been interpreted as the relaxation of the initially excited state through intramolecular charge transfer (ICT) mechanism. Excited state intramolecular proton transfer reactions of coumarin derivatives have also been reported in homogenous media. Coumarin 4 was found to exhibit ESPT in water and in binary solvent mixtures. ${ }^{13}$ Coumarin 4 behaved as a photoacid in aqueous solution at neutral $\mathrm{pH}$ delivering a proton to the solvent. But in acidic $\mathrm{pH}$, it acts as a photobase accepting a proton from the solvent. ESPT behaviour of various aminocoumarins are also investigated in various polar solvents. ${ }^{14}$ Coumarin 102 is also being reported to show excited state proton transfer (ESPT) reactions in aqueous ${ }^{15}$ and alcoholic medium ${ }^{16}$ on addition of acids. The proton transfer takes place from the solvent via diffusive process. In this article, we report the ESPT investigations of C-102 in micro-heterogeneous media. The motivation for this study is two-fold: First, the ability of the micro-heterogenous media such as sodium dodecyl sulfate (SDS) micelles to enhance the ESPT reaction and second to be able to explore and quantify the local $\mathrm{pH}$ at water interface of SDS micelles. 


\section{Experimental}

\subsection{Preparation of the sample}

Sodium dodecyl sulfate (SDS) was obtained from Sigma-Aldrich and was used without further purification. Stock solution of SDS was prepared which was diluted to prepare SDS solution of varying concentrations. SDS solution of $25 \mathrm{mM}$ was used for all the fluorescence studies with different acids using double distilled water. Salicylic acid (SA) obtained from Aldrich was used as received. Stock solution SA of concentration of $200 \mathrm{mM}$ was prepared in methanol and required volumes were added to the SDS solution to obtain the desired concentration of SA. Concentrated hydrochloric acid obtained from Merck was diluted to the required concentrations. Laser grade coumarin-102 was obtained from Radiant dyes was used as received.

\subsection{Steady-state absorption and fluorescence measurements}

Absorption spectra for all the samples were recorded with Jasco V-530 UV-visible spectrophotometer. Steady state fluorescence spectra were recorded in Varian CARY Eclipse Fluorescence spectrophotometer. The excitation wavelength $\left(\lambda_{\text {ex }}\right)$ is $395 \mathrm{~nm}$. The absorbance at this wavelength is kept low to avoid inner filter effects.

\subsection{Time-resolved fluorescence measurements}

Time-resolved fluorescence measurements are performed at the magic angle of $54.7^{\circ}$ using a picosecond pulsed diode laser based TCSPC fluorescence spectrometer with $\lambda_{\text {ex }}$ at $406 \mathrm{~nm}$ from IBH, UK. The emission is collected at a right angle to the direction of the excitation beam at wavelength 480 and $560 \mathrm{~nm}$. The full width at half-maximum of the instrument response function was $98 \mathrm{ps}$ for $377 \mathrm{~nm}$ laser excitation. The resolution used for the present measurements was $14.1 \mathrm{ps} / \mathrm{channel}$. The data are fitted to multiexponential functions after deconvolution of the instrument response function by an iterative reconvolution technique using the IBH DAS 6.2 data analysis software, a procedure based on Marquardt's algorithm. The reduced $\chi^{2}$ values close to 1 and the weighted residuals serve as parameters for goodness of fit.

\subsection{Computational details}

The proton affinities of various proton binding sites of coumarin-102 was calculated at the B3LYP level of theory using $6-311++\mathrm{G}^{*}$ basis set for both the ground state as well as the first excited state using Gaussian 03 suite of programs. ${ }^{17}$

\section{Results and discussion}

The steady state absorption spectrum of C-102 in SDS micelles shows a single maximum at $397 \mathrm{~nm}$ (figure not shown), which is in good agreement with the results reported in the literature. ${ }^{10}$ Addition of acids, such as hydrochloric acid and salicylic acid to the above system, does not alter the absorption spectrum and clearly indicates the presence of single absorbing species in the ground state. The steady state emission spectra of C-102 in SDS micelles show a single broad band with the maximum around $480 \mathrm{~nm}$, which once again is in excellent agreement with the spectrum reported earlier. ${ }^{18} \mathrm{On}$ addition of either hydrochloric acid $(\mathrm{HCl})$ or salicylic acid (SA) to the SDS micellar solution containing C-102, resulted in emergence of new red shifted band with maximum around $530 \mathrm{~nm}$, as depicted in figure 1. In figure 1, all the emission spectra were area normalized to remove any error arising from minor difference in concentration of the dyes. ${ }^{19}$ The intensity of the newly appearing band around $530 \mathrm{~nm}$ increased with increasing concentration of $\mathrm{HCl} / \mathrm{SA}$ at the expense of the original band at $478 \mathrm{~nm}$. The enhancement of $530 \mathrm{~nm}$ band was higher upon addition of $\mathrm{HCl}$ in comparison with addition of identical amounts of SA. This can be attributed to the difference in the degree of dissociation of the two acids. The area normalized steady state emission spectra (see figure 1) clearly show an isoemissive point at $510 \mathrm{~nm}$, which indicates the presence of two distinct emissive species originating out of single initially excited state. ${ }^{19}$ It was also observed that at very high concentrations of $\mathrm{HCl}$ (about $10 \mathrm{mN}$ ), the $480 \mathrm{~nm}$ band disappear, almost completely, in favour of the $530 \mathrm{~nm}$ band. Similar investigations carried out in cetyltrimethylammonium bromide (CTAB) and Triton-X 100 micelles in the presence of varying concentrations of $\mathrm{HCl}$ and $\mathrm{SA}$, resulted in a simple emission spectrum with peak around $480 \mathrm{~nm}$ with the conspicuous absence of any emission band around $525-530 \mathrm{~nm}$. The emergence of this red-shifted peak is being attributed to excited state proton transfer (ESPT) behaviour of aminocoumarins as reported earlier by 

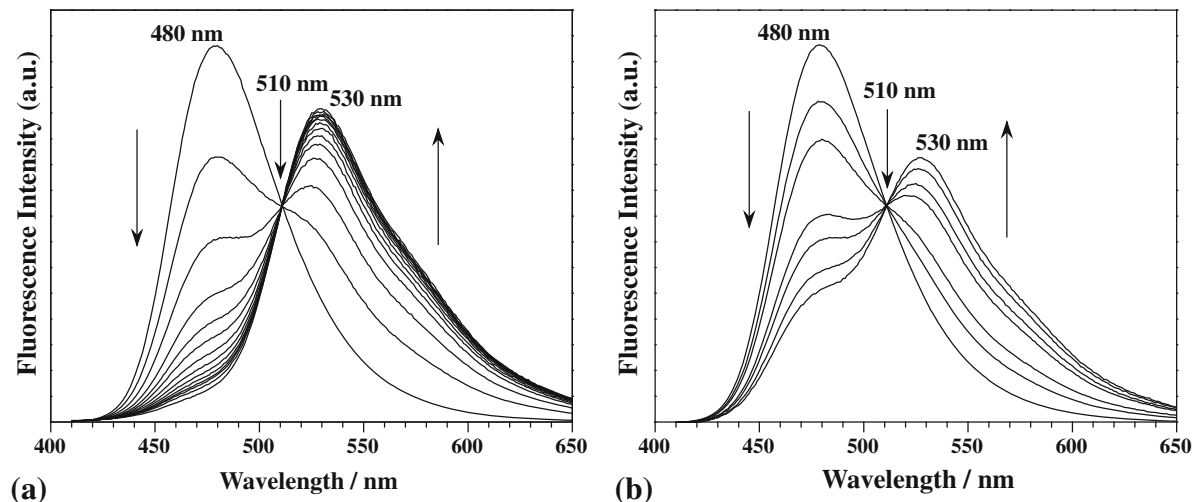

(b)

Figure 1. Steady state emission spectra of C-102 in SDS micelles with varying concentrations of (a) $\mathrm{HCl}(0,1,2,3,4,5,6,7,8,9$ and $10 \mathrm{mN})$ and (b) $\mathrm{SA}(0,0.5,1,3,5$, 7 and $10 \mathrm{mM}$ ). The direction of arrows indicates the increase in concentration of $\mathrm{HCl}$ in (a) and increase in concentration of SA in (b).

Campillo et al. ${ }^{15}$ However, in order to completely eliminate the possibility of the presence of multiple species in ground state, we had also carried out red edge excitation shift spectra. The invariance of the emission spectra upon excitation at various positions in the absorption band clearly points out the presence of single absorbing species in the present system. Coupled with the fact that addition of both $\mathrm{HCl}$ and SA lead to the appearance of new band, it can be interpreted as formation of an excited state protonated species of C-102 upon excitation. Formation of such species is facilitated by the presence of availability of the protons in the solution. This observation is in contrast with the red-edge excitation shift spectra observed in case of coumarin-153 in bile salt (sodium deoxycholate) aggregate, wherein the emission spectra showed remarkable dependence on the excitation wavelength, resulting in a marked change in emission intensities of the two peaks originating from two distinct species in different environments. ${ }^{20}$

The time resolved fluorescence measurements were carried out for C-102 in SDS micelles without and with the presence of acids such as $\mathrm{HCl}$ and $\mathrm{SA}$ in varying concentrations. Figure 2 presents representative decay curves collected at 480 and $560 \mathrm{~nm}$ for the C-102 in SDS micelles and with the presence of $1 \mathrm{mN} \mathrm{HCl}$ and $10 \mathrm{mM} \mathrm{SA}$. The fluorescence decay curves were recorded at $560 \mathrm{~nm}$, under the assumption that at this wavelength only the protonated excited state is sampled exclusively and the contribution from the emission of the initially excited state is almost negligible. The decay of C-102 in SDS micellar medium at $480 \mathrm{~nm}$ was found to be single exponential with a lifetime of $6.09 \mathrm{~ns}$ which is in good agreement with the value reported in the literature. ${ }^{21}$ Further, the fluorescence decay curve recorded

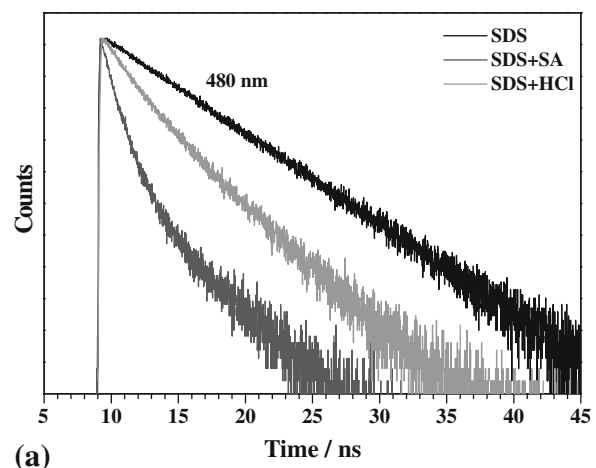

(a)

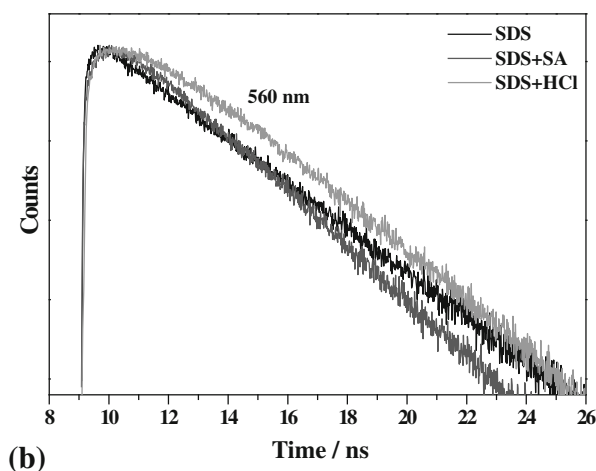

(b)

Figure 2. Time resolved fluorescence decay curves C-102 in SDS micelles and with the addition of (a) $10 \mathrm{mM} \mathrm{SA}$ and (b) $1 \mathrm{mN} \mathrm{HCl}$ recorded with excitation at $406 \mathrm{~nm}$ and emission monitored at (a) $480 \mathrm{~nm}$ and (b) $560 \mathrm{~nm}$. 
at $560 \mathrm{~nm}$ could be fitted to a biexponential function with a rise time of $0.31 \mathrm{~ns}$, which can be attributed to the solvation dynamics of C-102 in SDS micelles. ${ }^{18}$ In the presence of the $1 \mathrm{mN} \mathrm{HCl}$ the decay of $\mathrm{C}-102$ in SDS medium at $480 \mathrm{~nm}$ can be fitted to biexponential function with lifetimes of 1.23 and $3.27 \mathrm{~ns}$, with the corresponding amplitudes being 0.58 and 0.42 , respectively. The decay curve collected at $560 \mathrm{~nm}$ can also be fitted to a biexponential function, however, the amplitude of one of the exponents is negative, which indicates the growth kinetics for the formation of a protonated state, the protonated state then decays with a fluorescence lifetime of $5.53 \mathrm{~ns}$. It is interesting to note that the faster lifetime of $1.23 \mathrm{~ns}$ present in the fluorescence decay curve recorded at $480 \mathrm{~nm}$ matches exactly with the raise-time of $1.23 \mathrm{~ns}$ present in the fluorescence decay curve recorded at $560 \mathrm{~nm}$. Therefore, the lifetime of $1.23 \mathrm{~ns}$ present in $480 \mathrm{~nm}$ fluorescence decay curve can be attributed to the decay of the initially excited state leading to formation of a protonated state while the lifetime of $3.27 \mathrm{~ns}$ is the decay of the initially excited state. The difference in the lifetimes of the decay of the initially excited state to the ground state in the absence (6.09 ns) and in the presence $(3.27 \mathrm{~ns})$ of $\mathrm{HCl}$ can be attributed to the change in the environment due to the addition of $\mathrm{HCl}$. The decrease in lifetime of the initially excited state from 6.09 to $3.27 \mathrm{~ns}$ is due to enhanced quenching of fluorescence resulting due to the presence of the acid. Similar observations were made in the presence of $10 \mathrm{mM} \mathrm{SA}$. In this case the fluorescence decay at $480 \mathrm{~nm}$ was characterized by the presence of two distinct lifetimes of 0.72 and $2.1 \mathrm{~ns}$ with relative amplitudes of 0.72 and 0.28 , respectively. On the other hand, the fluorescence decay curve at $560 \mathrm{~nm}$ once again shows the presence of growth kinetics for the formation of the protonated state with lifetime of $0.73 \mathrm{~ns}$, which then decays with radiative lifetime of $5.03 \mathrm{~ns}$. Once again, in this case decay constant of $0.72 \mathrm{~ns}$ present in the fluorescence decay curve recorded at $480 \mathrm{~nm}$ matches well with the raisetime of $0.73 \mathrm{~ns}$ present in the fluorescence decay curve recorded at $560 \mathrm{~nm}$.

In order to further establish the relationship between the decay constant at $480 \mathrm{~nm}$ and the raise time at $560 \mathrm{~nm}$, the time-resolved fluorescence decay curves of C-102 in SDS micelles in the presence of varying concentrations of $\mathrm{HCl}$ and SA were recorded. Tables 1 and 2 list all the lifetimes and their relative amplitudes measured at 480 and $560 \mathrm{~nm}$. The fluorescence decay curves at $480 \mathrm{~nm}$ were fitted to biexponential function. It can be seen from table 1 that increase in the concentration of $\mathrm{HCl}$ leads to decrease in the lifetime of the faster component along with increase in the corresponding amplitude. The fluorescence decay curves measured at $560 \mathrm{~nm}$ were also fitted to a biexponential function, however, one of the exponents carries a negative amplitude, which correspond to the growth of the protonated state. The growth (life) time decreases with increase in the $\mathrm{HCl}$ concentration, while the corresponding amplitude increases. The shorter lifetime decay component at $480 \mathrm{~nm}$ remains comparable to the rise time component at $560 \mathrm{~nm}$ in the entire concentration range. This close correlation between faster decay component at $480 \mathrm{~nm}$ with the rise time at $560 \mathrm{~nm}$ clearly points out that one of the channels of decay of the initially excited states leads to the formation of the protonated state. The protonated state then decays with the time constant of $\sim 5.2 \mathrm{~ns}$, and shows weak dependence on the $\mathrm{HCl}$ concentration. Similar observations can also be made for the concentration dependence of SA (see table 2).

Table 1. Time resolved fluorescence measurement data of C-102 in SDS micelles with varying $\mathrm{HCl}$ concentrations at $480 \mathrm{~nm}$ and $560 \mathrm{~nm}$.

\begin{tabular}{|c|c|c|c|c|c|c|c|c|}
\hline \multirow[t]{2}{*}{$\mathrm{HCl}$ conc. / $\mathrm{mN}$} & \multicolumn{4}{|c|}{$480 \mathrm{~nm}$} & \multicolumn{4}{|c|}{$560 \mathrm{~nm}$} \\
\hline & $\mathrm{a}_{1}$ & $a_{2}$ & $\tau_{1} / \mathrm{ns}$ & $\tau_{2} / \mathrm{ns}$ & $a_{1}$ & $a_{2}$ & $\tau_{1} / \mathrm{ns}$ & $\tau_{2} / \mathrm{ns}$ \\
\hline 0 & 1 & - & 6.09 & - & -0.24 & 1.24 & 0.31 & 6.20 \\
\hline 1 & 0.58 & 0.42 & 1.23 & 3.27 & -0.87 & 1.87 & 1.23 & 5.53 \\
\hline 2 & 0.84 & 0.16 & 0.91 & 2.93 & -0.72 & 1.72 & 0.94 & 5.24 \\
\hline 3 & 0.90 & 0.10 & 0.71 & 3.1 & -0.69 & 1.69 & 0.76 & 5.19 \\
\hline 4 & 0.92 & 0.08 & 0.57 & 3.05 & -0.56 & 1.56 & 0.60 & 5.19 \\
\hline 5 & 0.94 & 0.06 & 0.48 & 3.89 & -0.40 & 1.40 & 0.49 & 5.17 \\
\hline 6 & 0.94 & 0.06 & 0.40 & 3.86 & -0.37 & 1.37 & 0.42 & 5.17 \\
\hline 7 & 0.94 & 0.06 & 0.35 & 3.93 & -0.45 & 1.45 & 0.38 & 5.17 \\
\hline 8 & 0.94 & 0.06 & 0.32 & 3.66 & -0.37 & 1.37 & 0.36 & 5.18 \\
\hline 9 & 0.94 & 0.06 & 0.30 & 3.88 & -0.41 & 1.41 & 0.31 & 5.16 \\
\hline 10 & 0.94 & 0.06 & 0.29 & 3.91 & -0.39 & 1.39 & 0.31 & 5.17 \\
\hline
\end{tabular}


Table 2. Time resolved fluorescence measurement data of C-102 in SDS micelles with varying concentrations of SA at $480 \mathrm{~nm}$ and $560 \mathrm{~nm}$.

\begin{tabular}{|c|c|c|c|c|c|c|c|c|}
\hline \multirow[t]{2}{*}{ SA conc. / mM } & \multicolumn{4}{|c|}{$480 \mathrm{~nm}$} & \multicolumn{4}{|c|}{$560 \mathrm{~nm}$} \\
\hline & $a_{1}$ & $a_{2}$ & $\tau_{1} / \mathrm{ns}$ & $\tau_{2} / \mathrm{ns}$ & $a_{1}$ & $a_{2}$ & $\tau_{1} / \mathrm{ns}$ & $\tau_{2} / \mathrm{ns}$ \\
\hline 0 & 1 & - & 6.09 & - & -0.24 & 1.24 & 0.31 & 6.20 \\
\hline 0.5 & 0.21 & 0.79 & 2.02 & 5.33 & -0.34 & 1.34 & 2.02 & 5.99 \\
\hline 1 & 0.25 & 0.75 & 1.73 & 4.89 & -0.50 & 1.50 & 1.75 & 5.87 \\
\hline 3 & 0.59 & 0.41 & 1.43 & 3.58 & -0.81 & 1.81 & 1.40 & 5.31 \\
\hline 5 & 0.70 & 0.30 & 0.96 & 2.77 & -0.74 & 1.74 & 0.98 & 5.19 \\
\hline 7 & 0.72 & 0.28 & 0.79 & 2.33 & -0.73 & 1.73 & 0.79 & 5.11 \\
\hline 10 & 0.72 & 0.28 & 0.72 & 2.10 & -0.81 & 1.81 & 0.73 & 5.03 \\
\hline
\end{tabular}

The ability of SDS to promote proton transfer in C102 was verified by step-wise addition of SDS to a solution containing $10 \mu \mathrm{M} \mathrm{C}-102$ along with $10 \mathrm{mM}$ $\mathrm{SA}$ and the results are presented in figure 3. At lower concentrations of SDS there is hardly any change in the emission spectrum, on the other hand, there is an abrupt appearance of the red shifted peak around $8 \mathrm{mM}$ concentration of SDS, which happens to be the critical micelle concentration (CMC) of SDS. Figure 3b depicts the plot of observed intensities of 480 and $525 \mathrm{~nm}$ for various concentrations of SDS in the presence of $10 \mathrm{mM} \mathrm{SA}$. The observed trend can be related to the ability of SDS to promote excited state protonation reaction only after the formation of micelles. Further, the time resolved fluorescence measurements for the same set of solutions were also carried out and the data are displayed in table 3. In the absence of SDS the fluorescence decay of C-102 both at 480 and $560 \mathrm{~nm}$ could be fitted to single exponential with time constant of about $5.8 \mathrm{~ns}$. Addition of SDS beyond $4 \mathrm{mM}$ leads to biexponential decay with a faster decay component at $480 \mathrm{~nm}$ and a corresponding raise at $560 \mathrm{~nm}$, which can be related to the protonation of C-102 in the excited state. However, it is interesting to note that the formation of the protonated form is most rapid at SDS concentration of $8 \mathrm{mM}$, which corresponds to CMC of SDS. Both the steady state and the time resolved data clearly indicate that formation of micelles promotes the excitation protonation reaction of $\mathrm{C}-102$.

To evaluate the extent of protonation in the absence of SDS micelles the steady state emission spectra of C-102 in aqueous solution upon step-wise addition of $\mathrm{HCl}$ were recorded and the results are presented in figure 4 . The spectra reveal that the red-shifted band begins to appear after the addition of $20 \mathrm{mN} \mathrm{HCl}$ and the emission band due to locally excited state completely disappears upon addition of $100 \mathrm{mN} \mathrm{HCl}$. On the assumption that $\mathrm{HCl}$ completely dissociates in aqueous solutions, these two limits corresponds to $\mathrm{pH}$ values of 1.7 and 1. The comparison of steady state
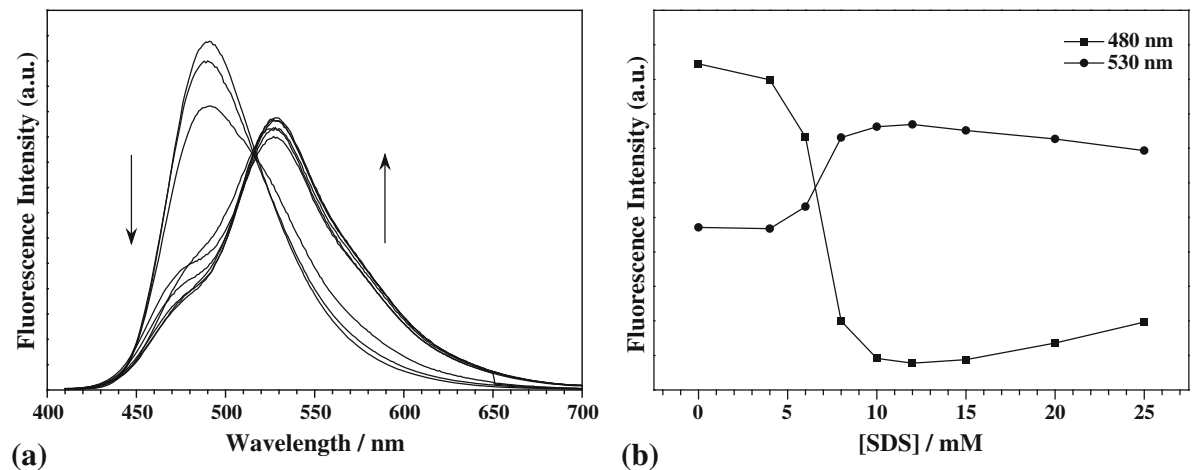

Figure 3. (a) Steady state emission spectra of C-102 with varying concentration of SDS (0 to $25 \mathrm{mM}$ ) in the presence of $10 \mathrm{mM}$ salicylic acid (SA) (direction of arrow indicates the increase in concentration of SDS). (b) Variation of fluorescence intensity at 480 and $530 \mathrm{~nm}$ with increase in concentration of SDS. 
Table 3. Time resolved fluorescence measurement data of C-102 at different SDS concentrations with addition of $10 \mathrm{mM}$ SA.

\begin{tabular}{|c|c|c|c|c|c|c|c|c|}
\hline \multirow[t]{2}{*}{ Conc. of SDS / mM } & \multicolumn{4}{|c|}{$480 \mathrm{~nm}$} & \multicolumn{4}{|c|}{$560 \mathrm{~nm}$} \\
\hline & $\mathrm{a}_{1}$ & $a_{2}$ & $\tau_{1} / \mathrm{ns}$ & $\tau_{2} / \mathrm{ns}$ & $a_{1}$ & $a_{2}$ & $\tau_{1} / \mathrm{ns}$ & $\tau_{2} / \mathrm{ns}$ \\
\hline 0 & 1.00 & - & 5.82 & - & 1.00 & - & 5.84 & - \\
\hline 4 & 1.00 & - & 4.33 & - & -0.25 & 1.25 & 0.94 & 4.61 \\
\hline 6 & 0.34 & 0.66 & 0.69 & 4.15 & -0.42 & 1.42 & 0.62 & 4.62 \\
\hline 8 & 0.82 & 0.18 & 0.40 & 4.15 & -0.96 & 1.96 & 0.40 & 4.96 \\
\hline 10 & 0.88 & 0.12 & 0.43 & 3.86 & -1.00 & 2.00 & 0.42 & 4.99 \\
\hline 12 & 0.91 & 0.09 & 0.48 & 3.74 & -0.90 & 1.90 & 0.48 & 5.04 \\
\hline 15 & 0.87 & 0.13 & 0.57 & 2.47 & -0.81 & 1.81 & 0.54 & 5.06 \\
\hline 20 & 0.89 & 0.11 & 0.62 & 2.70 & -0.81 & 1.81 & 0.61 & 5.07 \\
\hline 25 & 0.88 & 0.12 & 0.76 & 2.91 & -0.85 & 1.85 & 0.72 & 5.07 \\
\hline
\end{tabular}

emission spectra in the SDS micelles and aqueous solutions reveal that the excited state protonation occurs at much lower acid concentrations in the presence of SDS micellar medium. Fluorescence lifetimes were also recorded for C-102 in aqueous medium by addition of varying concentrations of $\mathrm{HCl}$. All the decays were fitted to a biexponential function and table 4 lists the relevant fluorescence lifetimes and their relative amplitudes. The decay measured at $480 \mathrm{~nm}$ had a faster component which has a close match with the rise time observed at $560 \mathrm{~nm}$ for all concentrations of $\mathrm{HCl}$. It can be seen from table 4 that the faster decay component at $480 \mathrm{~nm}$ and the corresponding rise time at $560 \mathrm{~nm}$ are lowered with the increase in concentration of protons. This can be interpreted as

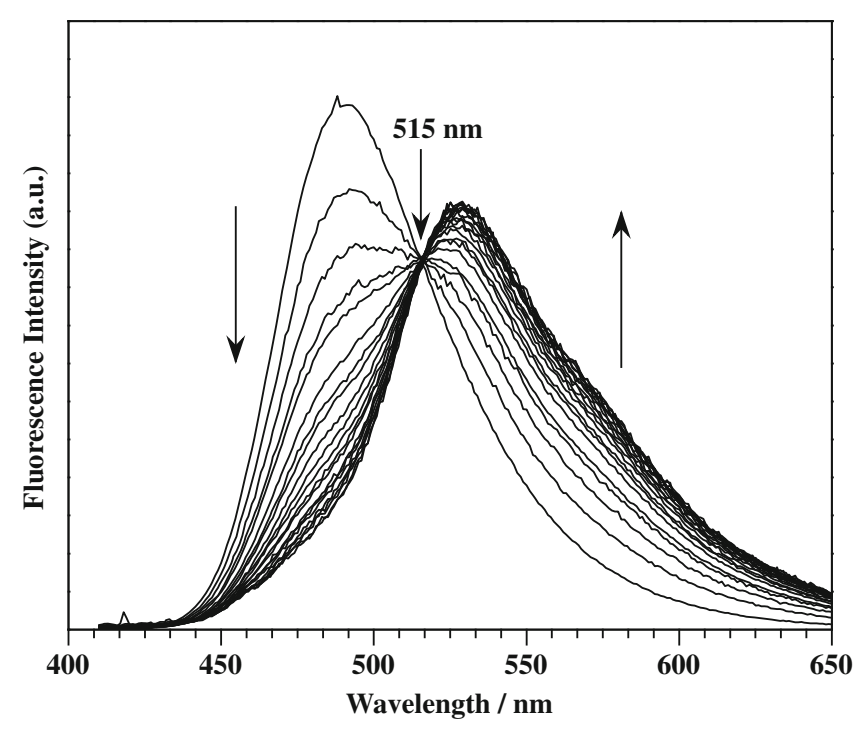

Figure 4. Steady state emission spectra of C-102 in aqueous medium with varying concentrations of $\mathrm{HCl}(0$ to $200 \mathrm{mN}$ ). Direction of arrow indicates the increase in concentration of $\mathrm{HCl}$. acceleration of the excited state protonation rate with increase in concentration of $\mathrm{HCl}$. It must be noted here that the fluorescence decay of $\mathrm{C}-102$ in aqueous medium at $560 \mathrm{~nm}$ showed a rise time $0.85 \mathrm{~ns}$ on addition of $30 \mathrm{mN} \mathrm{HCl}$ is comparable to the value obtained by addition of just $3 \mathrm{mN}$ of $\mathrm{HCl}$ in the presence of SDS micelles (a rise time of $0.82 \mathrm{~ns}$ ). The steady state spectra (figures 1a and 4) and the lifetime data (tables 1 and 4) clearly signify the fact that for a given concentration of acid, SDS micelles efficiently promotes the excited state portonation reaction in comparison to aqueous solutions.

The time-resolved area normalized emission spectra (TRANES) can confirm the existence of multiple emissive excited states arising from a single initially excited state. ${ }^{22}$ TRANES can clearly indicate the presence of two or more emissive species present in a system through the identification of iso-emmisive points. TRANES were calculated for C-102 in SDS micellar medium and also in the presence of $2 \mathrm{mN} \mathrm{HCl}$ and $10 \mathrm{mM}$ SA. The time-resolved emission spectra (TRES) were plotted after collecting decays between 450 and $540 \mathrm{~nm}$ with an interval of $5 \mathrm{~nm}$, which were area normalized to obtain the TRANES. The TRES were calculated from 0 to $5 \mathrm{~ns}$. The TRANES data for the C-102 in SDS micellar medium shifted continuously up to $300 \mathrm{ps}$ and with complete absence of iso-emissive point. This can be attributed to the solvation dynamics of C-102 in SDS micelles. ${ }^{22}$ In the presence of $2 \mathrm{mN} \mathrm{HCl}$, the TRANES data for the first $1100 \mathrm{ps}$ shows a shift in the emission maximum and with complete absence of iso-emissive point, which clearly indicates that solvation dynamics dominates in the this time period. However, the TRANES data for C-102 in SDS micelles in the presence of $2 \mathrm{mN} \mathrm{HCl}$ in the time period of $1100-2500 \mathrm{ps}$, depicted in figure $5 \mathrm{a}$, clearly shows the presence of an iso-emissive point at $508 \mathrm{~nm} .{ }^{11}$ The 
Table 4. Time resolved fluorescence measurement data of C-102 in aqueous medium with varying $\mathrm{HCl}$ concentrations at $480 \mathrm{~nm}$ and $560 \mathrm{~nm}$.

\begin{tabular}{|c|c|c|c|c|c|c|c|c|}
\hline \multirow[t]{2}{*}{$\mathrm{HCl}$ conc. / $\mathrm{mN}$} & \multicolumn{4}{|c|}{$480 \mathrm{~nm}$} & \multicolumn{4}{|c|}{$560 \mathrm{~nm}$} \\
\hline & $a_{1}$ & $a_{2}$ & $\tau_{1} / \mathrm{ns}$ & $\tau_{2} / \mathrm{ns}$ & $a_{1}$ & $a_{2}$ & $\tau_{1} / \mathrm{ns}$ & $\tau_{2} / \mathrm{ns}$ \\
\hline 10 & 0.21 & 0.79 & 1.27 & 4.14 & -0.43 & 1.43 & 1.30 & 4.32 \\
\hline 20 & 0.57 & 0.43 & 1.05 & 3.53 & -0.67 & 1.67 & 1.09 & 3.57 \\
\hline 30 & 0.70 & 0.30 & 0.90 & 3.39 & -0.59 & 1.59 & 0.85 & 3.39 \\
\hline 40 & 0.76 & 0.24 & 0.71 & 3.27 & -0.61 & 1.61 & 0.75 & 3.27 \\
\hline 50 & 0.81 & 0.19 & 0.58 & 3.19 & -0.52 & 1.52 & 0.59 & 3.23 \\
\hline 60 & 0.82 & 0.18 & 0.55 & 3.12 & -0.62 & 1.62 & 0.59 & 3.20 \\
\hline 70 & 0.84 & 0.16 & 0.47 & 3.10 & -0.57 & 1.57 & 0.50 & 3.17 \\
\hline 80 & 0.85 & 0.15 & 0.45 & 3.10 & -0.58 & 1.58 & 0.49 & 3.15 \\
\hline
\end{tabular}

TRANES measurements of C-102 in SDS micelles in the presence of $10 \mathrm{mM}$ of $\mathrm{SA}$, an iso-emissive point was observed at $508 \mathrm{~nm}$ in the time period of $800-3000 \mathrm{ps}$ (see figure $5 \mathrm{~b}$ ). The emergence of iso-emissive point at a later time in case of $2 \mathrm{mN} \mathrm{HCl}$ than in $10 \mathrm{mM} \mathrm{SA}$ is due to the slower formation time of the excited state as evident from the time-resolved fluorescent data (see tables 1 and 2).

It has been proposed in the literature that the species responsible for the emission band around $560 \mathrm{~nm}$ is the carbonyl protonated C-102. Scheme 1 illustrates the possible kinetic pathway. ${ }^{15}$ It is well-known that the excited state of $\mathrm{C}-102[\mathrm{C}]^{*}$ is much more polar due to charge separation, relative to the ground state [C]. The highly polar excited state undergoes protonation resulting in formation of $\left[\mathrm{CH}^{+}\right]^{*}$, which then radiatively decay with a lifetime of $\sim 5.2 \mathrm{~ns}$. The formation of $\left[\mathrm{CH}^{+}\right]^{*}$ from $[\mathrm{C}]^{*}$ is related to the concentration of the acid and most importantly the presence of SDS micelles. For instance, it can be seen that in aqueous solutions, the band at $480 \mathrm{~nm}$ disappeared almost completely upon addition of $\sim 100 \mathrm{mN}$ of $\mathrm{HCl}$, on the other hand, in the presence of SDS micelles addition of $10 \mathrm{mN}$ of $\mathrm{HCl}$ is adequate to show similar behaviour, which is a clear indication of the fact that SDS micelles promote the excited state protonation on C-102. These results can be interpreted in the light of the fact that the local $\mathrm{pH}$ at the micelle-water interface is lower than 7 due to the presence of higher negative charge density on the Stern layer of the SDS micelles. The positively charged hydronium ions migrate from the bulk water, thereby increasing the concentration of protons at the interface in comparison with the bulk water and can be substantial. It is well-known that C-102 preferentially resides at the interface and thus experiences a larger proton concentration. ${ }^{18}$ The larger effective concentration of protons at the surface micelles promotes the excited state protonation reactions in the presence of the SDS micelles.

Computational studies were performed to derive the proton affinities of various proton binding sites of coumarin-102 was calculated in both ground state and excited state at B3LYP level of theory using 6$311++\mathrm{G}^{*}$ basis set. From the calculated values (see
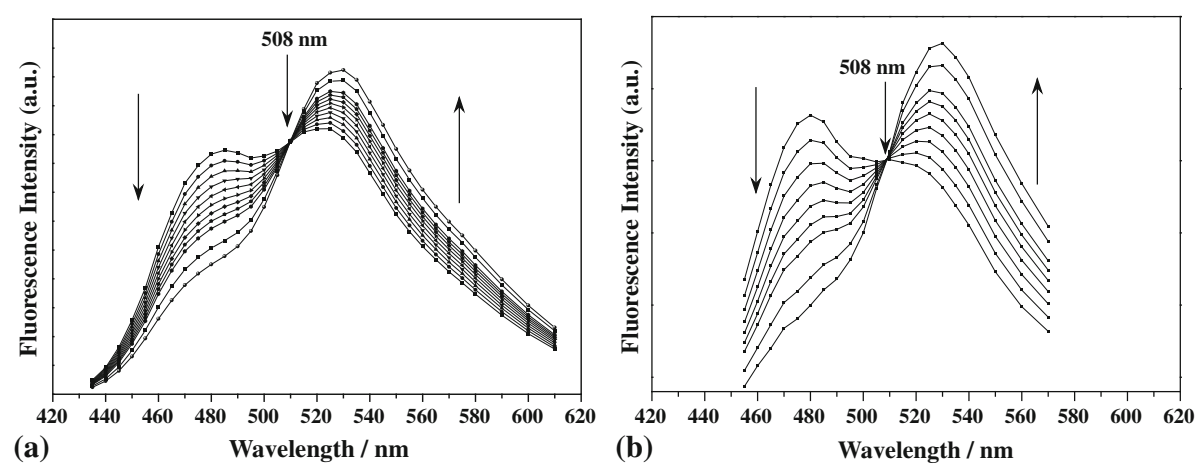

Figure 5. Time resolved area normalized emission spectra (TRANES) of C-102 in SDS micellar medium with addition of (a) $2 \mathrm{mN} \mathrm{HCl}$ in the time window 1100 $2500 \mathrm{ps}$ and (b) $10 \mathrm{mM} \mathrm{SA}$ in the time window 800-3000 ps. Direction of arrow indicates the increase in time. 


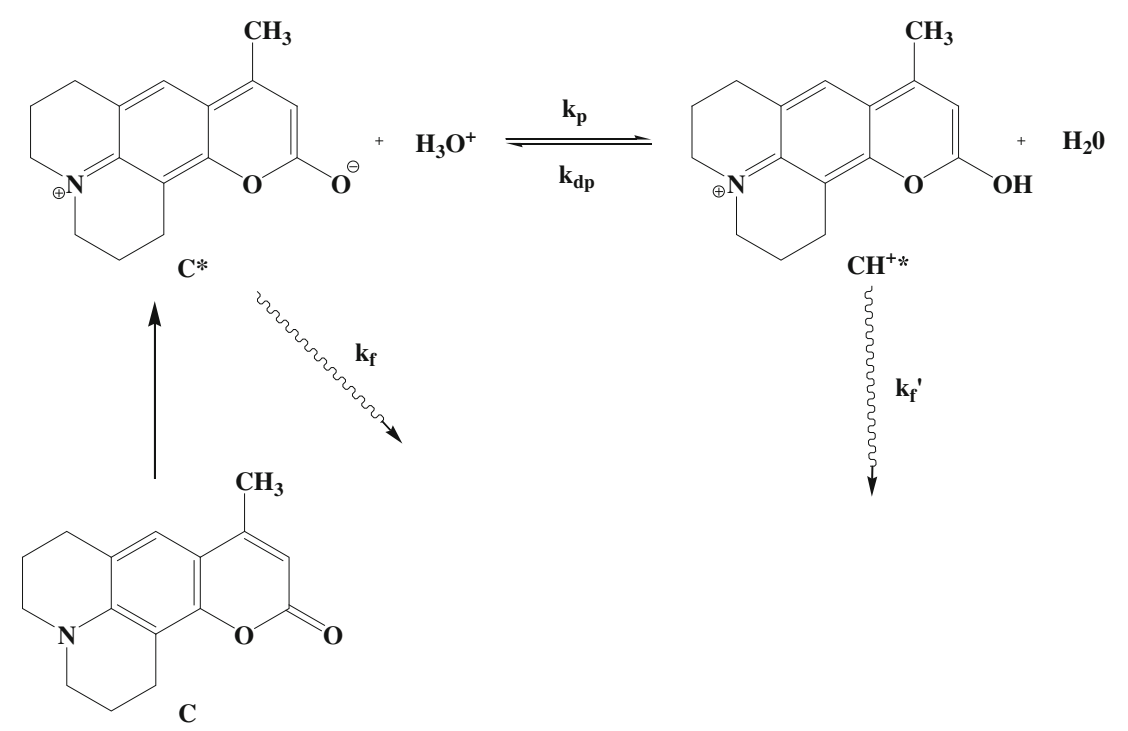

Scheme 1. Excited state proton transfer (ESPT) mechanism of Coumarin102 upon photoexcitation leading to formation of the excited species $\left(\mathrm{C}^{*}\right)$ and the protonated species in excited state $\left(\mathrm{CH}^{+*}\right)$ and their consequent relaxation through fluorescent emission.

table 5), it can be clearly seen that the most preferential site for proton binding is carbonyl oxygen followed by the tertiary amine nitrogen in the ground state. In the first electronically excited state, the carbonyl oxygen still has the highest proton affinity. Surprisingly, the amine nitrogen has the lowest proton affinity in the excited state, which is quite contradictory to the sequence of the ethereal oxygen comes next in the order. The calculated values predicts that the carbonyl oxygen will be protonated in the excited state which is in accordance with the proposed scheme. Additionally, there is a significant increase in the proton affinity values in the excited state compared to ground state which justifies the occurrence of protonation in the excited state.

The concentration on protons at the surface of a SDS micelle has been a subject of interest. The comparison of both relative intensities of the bands at 480 and $530 \mathrm{~nm}$ in the steady state emission spectra and also the kinetics of formation of $\left[\mathrm{CH}^{+}\right]^{*}$ from $[\mathrm{C}]^{*}$ can be used as reliable experimental parameter to estimate the local concentrations of protons at the SDS micelles interface. A closer look at steady state spectra shown in figures 1 and 4 reveals that the extent of protonation of is almost similar magnitude in the presence of $4 \mathrm{mN}$ $\mathrm{HCl}$ in SDS solutions and $60 \mathrm{mN} \mathrm{HCl}$ in aqueous solutions. Additionally, one can also see from tables 1 and 4 that in the presence of $4 \mathrm{mN} \mathrm{HCl}$ in SDS solutions the faster component of $[\mathrm{C}]^{*}$ decays with lifetime of $0.54 \mathrm{~ns}$, while the corresponding value in $60 \mathrm{mN} \mathrm{HCl}$ in aqueous solutions is 0.55 ps. Similarly, both the lifetime data corroborates well with the steady state spectra. Based on these observations it can be inferred that the local concentration of protons at the interface of SDS micelles is about a factor of 10-15. Earlier reports in the literature states that the concentration of protons on the surface of negatively charged SDS micelles is 80fold corresponding to that in bulk water. ${ }^{23}$ However, our results give a better estimation of the local concentration of protons at the SDS micellar surface because the fluorescent behaviour of the dye C-102 which resides in the Stern layer of SDS micelles is quite sensitive to

Table 5. Proton affinity $\left(\mathrm{kJ} \mathrm{mol}^{-1}\right)$ of various binding sites of coumarin 102 obtained by B3LYP/6-311++G*.

\begin{tabular}{lcc}
\hline & Ground state & First excited state \\
\hline Amine N & 924.8 & 874.4 \\
Ethereal O & 874.7 & 911.3 \\
Carbonyl O & 962.9 & 1018.8 \\
\hline
\end{tabular}



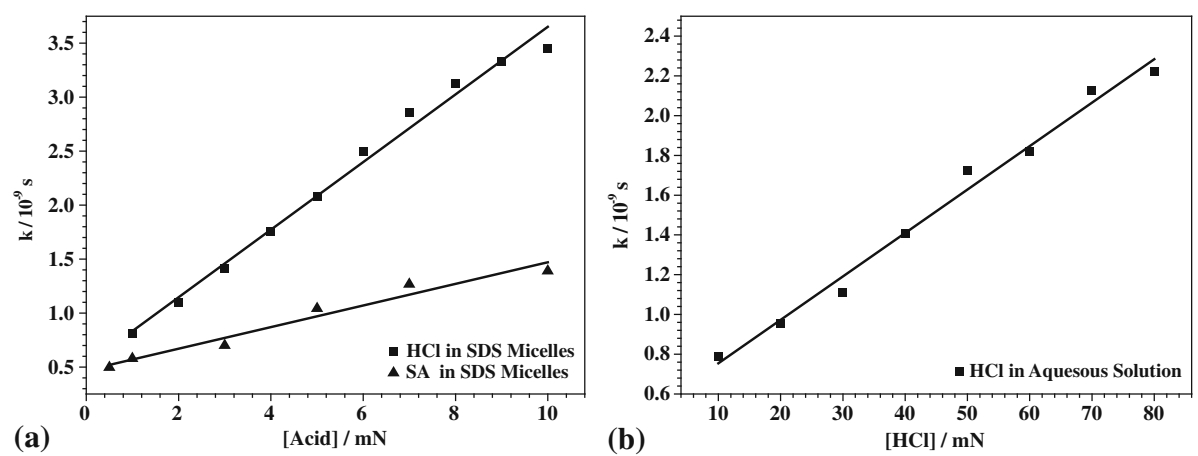

Figure 6. Plot of protonation rate $(k)$ versus the concentration of acid (a) in SDS

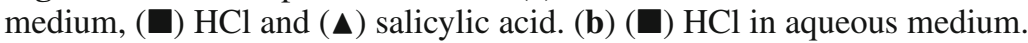

the surrounding microenvironment. A more quantitative approach to obtain the local concentration of protons on the micelle-water interface would be the solution of the following rate equation,

$$
k=k_{p}\left[H^{+}\right]_{\text {local }},
$$

where $k$ is the rate of the protonation reaction, $k_{p}$ is the protonation rate constant and $\left[\mathrm{H}^{+}\right]_{\text {local }}$ is concentration of protons at the micelle water interface. The protonation rate constant $k_{p}$ can be obtained from the slope of plot of protonation rate $(k)$ against the concentration of acid in aqueous medium in the absence of SDS micelles. The local concentration of protons,

$$
\left[H^{+}\right]_{\text {local }}=g \cdot\left[H^{+}\right]
$$

where $\left[\mathrm{H}^{+}\right]$is concentration of protons in the bulk water and ' $g$ ' is the enhancement factor. Substituting the value of $\left[\mathrm{H}^{+}\right]_{\text {local }}$ in equation 1 ,

$$
k=k_{p} \cdot g \cdot\left[H^{+}\right] \text {. }
$$

The slope of the plot of protonation rate $(k)$ against concentration of acid in micellar medium gives the value of ' $k_{p} . g$ ', as $k_{p}$ is known from the slope in aqueous medium, the enhancement factor ' $g$ ' can be easily calculated. The plot of protonation rate $(k)$ against concentration of acids in SDS micelles and aqueous medium is displayed in figure 6 . The value of protonation rate constant $\left(k_{p}\right)$ obtained from the slope of the plot in figure $6 \mathrm{~b}$ is $2.2 \times 10^{10} \mathrm{~mol}^{-1} \mathrm{dm}^{3} \mathrm{~s}^{-1}$ which is in the same order of magnitude as reported earlier. ${ }^{15}$ The enhancement factor ' $g$ ' was calculated from the slope of the plots in figure 6a. Thus, the enhancement factor ' $g$ ' which gives the measure of the local concentration of protons in micelle-water interface for $\mathrm{HCl}$ was found to be 14.3 and 4.6 for salicylic acid. The value of enhancement factor is remarkably low in case of SA compared to $\mathrm{HCl}$ as the factor of degree of dissociation of $\mathrm{SA}$ is also associated with it. Considering $\mathrm{HCl}$ to be completely ionized in aqueous medium, a better estimation of local concentration of proton at the micelle-water interface can be obtained. Thus from the enhancement factor, it can be concluded that the local concentration of protons in the micelle-water interface is 14.3 times more than in bulk water.

\section{Conclusion}

Coumarin-102 was found to behave as a photobase and show an enhancement in ESPT on addition of acids in SDS micellar medium compared to aqueous medium. This phenomenon was found to be absent in CTAB and Triton-X 100 micellar medium. So, this enhancement is attributed to the higher acidity in the SDS micellewater interface than in the bulk water. From the plot of protonation rate of $\mathrm{C}-102$ against the concentration of acid, the acidity at the micelle-water interface was found to be 14.3 times higher than that in the bulk water. The rate of the protonation reaction can be ascertained from the rise time observed in the time resolved fluorescence measurements which was found to be accelerated by increase in acidity of the medium. The steady state and the TRANES data are in compliance with the mechanism proposed in scheme 1 .

\section{Acknowledgements}

Authors thank Prof. A Dutta, Prof. A Samanta and Dr. H Pal for their help rendered during experiments and for stimulating discussions. Authors also wish to thank Mr. Susanta Halder for his help with computations. AD thanks Council of Scientific and Industrial Research, New Delhi (CSIR) for junior research fellowship. 


\section{References}

1. (a) Choi K and Hamilton A D 2001 Angew. Chem. Intl. Ed. 40 3912; (b) Chou P, McMorrow D, Aartsma T J and Kasha M 1984 J. Phys. Chem. 884596

2. (a) Poizat O, Bardez E, Buntinx G and Alain V 2004 J. Phys. Chem. A 108 1873; (b) Kim T G and Topp M R 2004 J. Phys. Chem. A 108 10060; (c) PérezLustres J L, Rodriguez-Prieto F, Mosquera M, Senyushkina T A, Ernsting N P and Kovalenko S A 2007 J. Am. Chem. Soc. 1295408

3. (a) Gíestas L, Chang Y, Lima J C, Vautier-Giongo C, Lopes A, Macanita A L and Quina F H 2003 J. Phys. Chem. A 107 3263; (b) Sarkar N, Das K, Das S, Datta A, Nath D N and Bhattacharyya K 1995 J. Phys. Chem. 99 17711; (c) Roy D, Karmakar R, Mondal S K, Sahu K and Bhattacharyya K 2004 Chem. Phys. Lett. 399 147; (d) Vautier-Giongo C, Chang Y, Moreina P F Jr, Lima J C, Fritas A A, Alver M, Quina F H and Macanita A L 2002 Langmuir 1810109

4. (a) Rath M C, Palit D K and Mukherjee T 1998 J. Chem. Soc. Faraday Trans. 94 1189; (b) Organero J A, Tormo L and Douhal A 2002 Chem. Phys. Lett. 363 409

5. Dutta P, Halder A, Mukherjee S, Sen P, Sen S and Bhattacharyya K 2002 Langmuir 187867

6. Mukherjee T K, Ahuja P, Koner A L and Datta A 2005 J. Phys. Chem. B 10912567

7. Fukuda M, Terazima M and Kimura Y 2008 Chem. Phys. Lett. $\mathbf{4 6 3} 364$

8. (a) Kunjappu J T 1993 J. Photochem. Photobiol. A: Chemistry 71 269; (b) Jones II G, Jackson W R and Choi C Y 1985 J. Phys. Chem. 89294

9. Seth D, Chakraborty A, Setua P, Chakrabarty D and Sarkar N 2005 J. Phys. Chem. B 10912080

10. Tamoto Y, Segawa H and Shirota H 2005 Langmuir 21 3757

11. (a) Chandrika P, Kulatilleke A, Saliya A, de Silva B and Eliav Y 2006 Polyhedron 25 2593; (b) Kim T K, Lee D N and Kim H J 2008 Tet. Lett. 494879
12. Barik A, Kumbhakar M, Nath S and Pal H 2005 Chem. Phys. 315277

13. Cohen B and Huppert D 2001 J. Phys. Chem. A 1057157

14. Bursulaya B D, Druzhinin S I and Uzhinov B M 1995 J. Photochem. Photobiol. A: Chemistry 9053

15. Campillo A J, Clark J H, Shapiro S L, Winn K R and Woodbridge P K 1979 Chem. Phys. Lett. 67218

16. Bursulaya B D, Druzhinin S I and Uzhinov B M 1995 J. Photochem. Photobiol. A: Chemistry 92163

17. Frisch M J, Trucks G W, Schlegel H B, Scuseria G E, Robb M A, Cheeseman, J R, Montgomery J A Jr, Vreven T, Kudin K N, Burant J C, Millam J M, Iyengar S S, Tomasi J, Barone V, Mennucci B, Cossi M, Scalmani G, Rega N, Petersson G A, Nakatsuji H, Hada M, Ehara M, Toyota K, Fukuda R, Hasegawa J, Ishida M, Nakajima T, Honda Y, Kitao O, Nakai H, Klene M, Li X, Knox J E, Hratchian H P, Cross J B, Bakken V, Adamo C, Jaramillo J, Gomperts R, Stratmann R E, Yazyev O, Austin A J, Cammi R, Pomelli C, Ochterski J W, Ayala P Y, Morokuma K, Voth G A, Salvador P, Dannenberg J J, Zakrzewski V G, Dapprich S, Daniels A D, Strain M C, Farkas O, Malick D K, Rabuck A D, Raghavachari K, Foresman J B, Ortiz J V, Cui Q, Baboul A G, Clifford S, Cioslowski J, Stefanov B B, Liu G, Liashenko A, Piskorz P, Komaromi I, Martin R L, Fox D J, Keith T, Al-Laham M A, Peng C Y, Nanayakkara A, Challacombe M, Gill P M W, Johnson B, Chen W, Wong M W, Gonzalez C and Pople J A 2003 Gaussian 03, Revision A.1 (Wallingford, CT: Gaussian, Inc.)

18. Sarkar N, Datta A, Das S and Bhattacharyya K 1996 J. Phys. Chem. 10015483

19. Panda D and Datta A 2008 Appl. Spec. 62341

20. Mandal U, Ghosh S, Das D K, Adhikari A, Dey S and Bhattacharyya K 2008 J. Chem. Sci. 12015

21. Kumbhakar M, Singh P K, Nath S, Bhasikuttan A C and Pal H 2008 J. Phys. Chem. B 1126646

22. Koti A S R, Krishna M M G and Periasamy N 2001 J. Phys. Chem. A 1051767

23. Lima C J, Vautier-Giongo C, Lopes A, Melo E, Quina F H and Macanita A L 2002 J. Phys. Chem. A 1065851 